\title{
Effect of Cold Pressor Test on the Internal Diameter of the Radial Artery
}

\author{
François Perret, Vincent Mooser, Bernard Waeber, Tardy Yanik, Meister Jean-Jacques,
} Emmanuel Mooser, Jürg Nussberger, and Hans R. Brunner

The aim of this study was to investigate in normal subjects the effect of a cold pressor test on the caliber of the radial artery, a muscular artery of medium size. The internal diameter of this artery was measured continuously using a recently developed ultrasonic device. Immersion of one hand in ice water for two minutes increased blood pressure from 115/75 $\pm 3 / 2$ (Mean \pm SEM) to 136/ $90 \pm 6 / 2 \mathrm{~mm} \mathrm{Hg}(P<.001)$ and decreased the internal diameter of the radial artery from $2.82 \pm$ 0.12 to $2.60 \pm 0.09 \mathrm{~mm}(P<.01)$. These data therefore indicate that the vasoconstriction induced by the cold pressor test involves not only arterioles, but also medium-size arteries. Am J Hypertens 1989; 2:727-728.

KEY WORDS: Echo-tracking system, servoplethysmomanometer, constriction of medium size arteries.
I mmersing a hand in ice water, the so-called "cold pressor test," causes in the majority of persons a rise in blood pressure and heart rate. ${ }^{1}$ The increase in vascular resistance induced by this maneuver has been attributed to a generalized sympathetic activation. Thus, the cold pressor test is known to heighten plasma norepinephrine levels ${ }^{2-4}$ and muscle sympathetic nerve activity. ${ }^{5}$ Smooth muscle cells are innervated by sympathetic fibers ubiquitously along the vascular tree. The blood pressure response to cutaneous application of ice water is probably due mainly to a contraction of arterioles, ie, of the blood vessels contributing the most to the regulation of peripheral resistance. The present study was undertaken to assess whether a medium-sized artery such as the radial artery also constricts during a cold pressor test. Our study involved normal subjects and used a new ultrasonic device that makes it possible to measure continuously and noninvasively the internal diameter of the radial artery throughout the cardiac cycle.

From the Division of Hypertension, University Hospital, Lausanne, Switzerland (FP, VM, BW, JN, HRB), and Department of Physics, Swiss Federal Institute of Technology, Lausanne, Switzerland (TY, MJJ, EM).

Address correspondence and reprint requests to Hans R. Brunner, MD, Division of Hypertension, C.H.U.V., 1011 Lausanne, Switzerland.

\section{METHODS}

The ultrasonic system used to locate the anterior and posterior wall of the radial artery consists of the following components: an emittor (Panametric, Model 5052 UA), a transducer (ASULAB SA, Research Laboratories of the SMH Group, Neuchâtel, Switzerland), a receptor (ASULAB), and an echo-tracking system (ASULAB). A flexible support (ASULAB) and a Doppler apparatus (Schiller Sonovit SV1, Basel, Switzerland) are utilized for positioning the transducer. The characteristics of the elements developed by ASULAB together with the Swiss Federal Institute of Technology will be described in detail below. Data are recorded and analyzed with a computerized acquisition system. The accuracy of this system has been tested by irradiating an aluminum target surface immersed in a tank of water, located at a distance equal to the focus of the transducer, and fixed perpendicularly to the ultrasonic beam. The depth of the aluminum plaque was modified with a micrometric screw. Changes in depth in the order of $0.001 \mathrm{~mm}$ could be detected. For a displacement of $1 \mathrm{~mm}$, the difference between depth changes derived from the micrometric scale and those based on the experimental system ranged between 0.001 and $0.002 \mathrm{~mm}$.

Healthy volunteers (seven men and three women) aged 15 to 27 years with a mean of 24 years, were studied in a supine position after a rest of at least 30 minutes. 


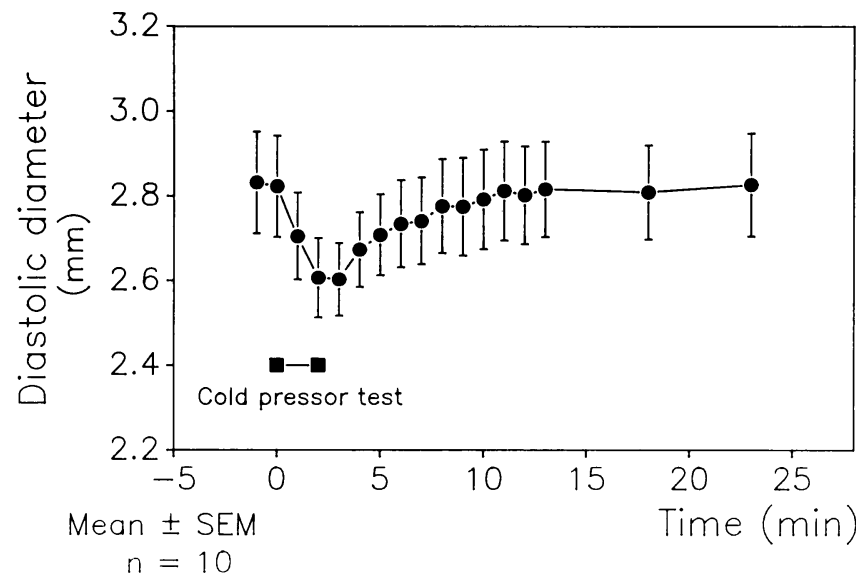

FIGURE 1. Effect of cold pressor test (controlateral hand in ice water for two minutes) on the diastolic diameter of the ipsilateral radial artery.

The transducer was placed over the radial artery, $4 \mathrm{~cm}$ above the major skin fold of the wrist. All readings were obtained on the right side, with the arm extended and fixed comfortably on a splint. Caution was used not to exert any pressure on the skin. A gel was applied between the extremity of the transducer and the skin surface. Blood pressure was measured continuously throughout the study using a servo-plethysmomanometer (Finapres, Ohmeda, Zug, Switzerland). ${ }^{6}$ This apparatus was fitted at the right median finger. The studied parameters were measured before, during a two-minute immersion of the subject's left hand (up to the wrist) in ice water, as well as during a 20 -minute recovery period.

A one-way analysis of variance was used for statistical evaluation of the results. This was followed by a least significant difference (LSD) test whenever a $P$ value of less than .05 was reached. Results are reported as mean \pm SEM.

\section{RESULTS}

During the two-minute cold pressor test, blood pressure of the ten subjects increased from $115 / 75 \pm 3 / 2$ to $136 / 90 \pm 6 / 2 \mathrm{~mm} \mathrm{Hg}(P<.001)$. Blood pressure levels returned to baseline values within five minutes after the end of the test.

The diastolic diameter of the radial artery decreased during the cold pressor test from $2.82 \pm 0.12$ to $2.60 \pm$ $0.09 \mathrm{~mm}(P<.01)$ and returned to control levels within ten minutes thereafter. No change in the difference between systolic and diastolic diameter was observed during the experiment.

\section{DISCUSSION}

An ultrasonic instrument was developed to measure noninvasively the internal diameter of peripheral arteries together with the diameter changes that occur with the pulse wave. With this device, the anterior and posterior wall of the radial artery, a muscular artery of medium size, can be continuously tracked.

In the present study, the increase in blood pressure that occurred during the cold pressor test was associated with a clear-cut reduction in the internal diameter of the radial artery. This finding indicates that the vasoconstriction resulting from cutaneous application of ice water is not limited to arterioles but also involves medium-sized arteries.

\section{REFERENCES}

1. Greene MA, Boltax AJ, Lustig GA, Rogow E: Circulatory dynamics during the cold pressor test. Am J Cardiol $1965 ; 16: 54-60$.

2. Cummings MF, Steele PM, Mahar LF, et al: The role of adrenal medullary catecholamine release in the response to a cold pressor test. Cardiovasc Res 1983;17:189-191.

3. Winer N, Carter C: Effect of cold pressor stimulation on plasma norepinephrine, dopamine- $\beta$-hydroxylase, and renin activity. Life Sci 1977;20:887-894.

4. Robertson D, Johnson GA, Robertson RM, et al: Comparative assessment of stimuli that release neuronal and adrenomedullary catecholamines in man. Circulation 1979;59:637-643.

5. Victor RG, Leimbach WN, Seals DR, et al: Effects of the cold pressor test on muscle sympathetic nerve activity in humans. Hypertension 1987;9:429-436.

6. Molhoek GP, Wesseling KH, Settels JJM, et al: Evaluation of the Penaz servo-plethysmo-manometer for the continuous, non-invasive measurement of finger blood pressure. Basic Res Cardiol 1984;79:598-609. 